\title{
Spinal arteriovenous shunts: accuracy of shunt detection, localization, and subtype discrimination using spinal magnetic resonance angiography and manual contrast injection using a syringe
}

\author{
Kittisak Unsrisong, MD, Siriporn Taphey, BS, and Kanokporn Oranratanachai, MD \\ Department of Radiology, Maharaj Nakorn Chiang Mai Hospital, Chiang Mai University, Chiang Mai, Thailand
}

\begin{abstract}
OBJECT The object of this study was to evaluate the accuracy of fast 3D contrast-enhanced spinal MR angiography (MRA) using a manual syringe contrast injection technique for detecting and evaluating spinal arteriovenous shunts (AVSs).

METHODS This was a retrospective study of 15 patients and 20 spinal MRA and catheter angiography studies. The accuracy of using spinal MRA to detect spinal AVS, localize shunts, and discriminate the subtype and dominant arterial feeder of the AVS were studied.
\end{abstract}

RESULTS There were 14 pretherapeutic and 6 posttherapeutic follow-up spinal MRA and catheter spinal angiography studies. The spinal AVS was demonstrated in 17 of 20 studies. Spinal MRA demonstrated $100 \%$ sensitivity for detecting spinal AVS with no false-negative results. A $97 \%$ accuracy rate for AVS subtype discrimination and shunt level localization was achieved using this study's diagnostic criteria. The detection of the dominant arterial feeder was limited to 9 of these 17 cases (53\%).

CONCLUSIONS The fast 3D contrast-enhanced MRA technique performed using manual syringe contrast injection can detect the presence of a spinal AVS, locate the shunt level, and discriminate AVS subtype in most cases, but is limited when detecting small arterial feeders.

http://thejns.org/doi/abs/10.3171/2015.7.SPINE15319

KEY WORDS spine; magnetic resonance angiography; spinal cord vascular diseases; arteriovenous fistula; vascular disorders

$\mathrm{S}$ PINAL arteriovenous shunt (AVS) is a rare condition with a variety of clinical presentations, from acute symptoms related to hematomyelia, spinal subarachnoid, or epidural hemorrhage to chronic progressive neurological symptoms related to spinal cord compression or congestive myelopathy. ${ }^{15,30}$ Spinal AVS can be either nidal or fistulous in terms of angioarchitecture, for which multiple classification schemes have been proposed. ${ }^{3,11,15,31,32,34}$ AVS can be classified into 4 groups based on their relationship with the spinal dura mater. Intradural shunts comprise AVSs within the spinal dura mater at the spinal cord, filum, or nerve roots. Dural and epidural AVSs are located at the level of the spinal dura mater and epidural space, respectively. Lastly, paraspinal AVS is a shunt located outside the spine. Diagnosing these diseases remains a challenging task, in which age, clinical presentation, and imaging findings are all needed to make the correct diagnosis. Currently, catheter spinal angiography is the gold-standard diagnostic tool for evaluating the cord supply, exact shunt location, and angioarchitectural features needed for therapeutic decision-making. ${ }^{3,11,32}$ Because of the long length of the spine and lack of image-based predictors of shunt location for non-nidal type spinal AVS, contrast-enhanced spinal MR angiography (MRA) is currently used as a preangiographic evaluation tool for differentiating the type of spinal AVS and determining the shunt location and dominant arterial feeder. One previous study has shown that spinal MRA can substantially reduce the radiation dose and the volume of iodinated contrast medium used in catheter spinal angiography.2. Many techniques for contrast-enhanced spinal MRA

ABBREVIATIONS AVM = arteriovenous malformation; $A V S$ = arteriovenous shunt; FOV = field of view; MIP = maximum intensity projection; MRA = MR angiography. SUBMITTED March 15, 2015. ACCEPTED July 23, 2015.

INCLUDE WHEN CITING Published online December 18, 2015; DOI: 10.3171/2015.7.SPINE15319. 
have been reported, all of which used an automatic contrast injector machine. Due to the small amount of contrast agent used in our institution's routine MRA protocol, we developed a simple contrast injection technique that uses a hand syringe in order to lower the cost of using the injector machine syringe, connecting tube, and Y-valve. Developing this manual technique led us to retrospectively review the accuracy of using contrast-enhanced spinal MRA with the hand syringe contrast injection protocol. The purpose of this study is to determine the accuracy of using contrastenhanced spinal MRA with the manual syringe contrast injection protocol to detect spinal AVS, localize shunts, and discriminate the subtype and dominant arterial feeder of the AVS.

\section{Methods}

\section{Participants}

This retrospective study received approval from our institutional ethical committee to evaluate all patients with the clinical symptoms and spinal MRI findings suspicious for spinal AVS who were referred to the Department of Radiology, Maharaj Nakorn Chiang Mai Hospital, between January 2010 and June 2014. The exclusion criterion was no available spinal MRA or catheter angiography for interpretation.

\section{MRA Acquisition Protocol}

All spinal MR angiograms were performed on a 1.5T MR imaging system (Signa Excite HD; GE Medical Systems) using an 8-channel cervicothoracolumbar spine array coil that included the entire spinal column and paraspinal area $1 \mathrm{~cm}$ lateral to the lateral foramen. The MRA protocol was a 3-phase, 3D, fast-spoiled gradient echopulse sequence with centric k-space filling, 400-mm field of view (FOV) in the craniocaudal direction, $512 \times 256$ matrix, 0.5-mm-thick sagittal sections, TR of $4.9 \mathrm{msec}$, TE of $1.4 \mathrm{msec}$, flip angle of $30^{\circ}$, and 40 - to 46 -second acquisition time for each phase. For spinal dural AVS cases, the initial MRA FOV was centered to include T-1 to L-5. If the shunting zone was not demonstrated, the FOV was shifted to include the cervical spine or sacrum depending on the location of the suspicious shunt on the earlier MR angiogram. No presaturation band was used. Intravenous access was obtained using an intravenous catheter no smaller than 20 gauge at the right antecubital vein. An 18-inch-long extension tube was used to connect the venous catheter to a 3-way Luer lock valve with a $20-\mathrm{ml}$ disposable syringe filled with contrast and saline. The contrast injection protocol consisted of hand syringe injection of $0.15 \mathrm{mmol} / \mathrm{kg}$ gadobenate dimeglumine (MultiHance) to a maximum volume of $20 \mathrm{ml}$, which was delivered at an approximate injection flow rate of $3 \mathrm{ml} / \mathrm{second}$, followed by $20 \mathrm{ml}$ of saline flush at the same rate. Contrast injection in all cases was performed by the same MR technician who was trained to control the injection rate to approximately $3 \mathrm{ml} / \mathrm{second}$ for adult cases and $2 \mathrm{ml} / \mathrm{second}$ for pediatric cases. MR fluoroscopy was used to determine the optimal scan delay, which was the time required to visualize the maximal enhancement of the entire aorta down to the aortic bifurcation.

\section{Image Postprocessing and Interpretation}

The MRA images were subtracted from the precontrast images on the AW workstation, and maximum intensity projection (MIP) images in the sagittal, coronal, and axial planes were created. Interpretation of each spinal MRA was made by agreement between 1 neuroradiologist and 1 neurointerventionist, both of whom have experience performing diagnostic catheter spinal angiography. The collected data included the detection of spinal AVS, subtype of spinal AVS, location of the shunt, and the dominant arterial feeder of the shunt. The criteria used for interpreting spinal MR angiograms included the enhancement and dilation of the spinal vein (either the intradural, epidural, or paraspinal vein) on the first phase of the spinal MRA (venous arterialization).

\section{Criteria Used for Subtype Discrimination \\ Paraspinal AVS}

Paraspinal AVS demonstrated an ectatic artery outside the spinal canal with arterialization of the paravertebral vein, with or without arterialization of the intradural perimedullary vein or epidural venous plexus..$^{10,14,32,35}$

\section{Spinal Dural AVS}

The ventral and dorsal epidural group demonstrated arterialization of the dilated spinal ventral or dorsal epidural venous plexus, with or without arterialization of the intradural perimedullary vein..$^{8,9,12,13,15,18,19,21,32}$ The lateral epidural group demonstrated arterialization of the intradural perimedullary vein in combination with hyperintense signal change of the spinal cord on the T2-weighted image and no arteriovenous malformation (AVM) nidus. $7,13,15,19,22,26,32$

\section{Intradural AVS}

The fistulous-type AVS demonstrated an enhanced dilated perimedullary vein or an ectatic venous pouch in combination with an enlarged spinal artery and no AVM nidus. ${ }^{7,11,15,20,32}$ The nidal-type AVS demonstrated a tangled tortuous vascular network of AVM nidus on or embedded within the spinal cord, conus, or nerve root. ${ }^{11,20,32}$

\section{Spinal Arteriovenous Metameric Syndrome}

Spinal arteriovenous metameric syndrome demonstrated multiple AVS locations with metameric links. ${ }^{32}$ Representative images of each spinal AVS subtype are shown in Figs. 1 and 2.

\section{Shunt Localization}

Paraspinal and intradural AVSs were determined by identifying the vertebral level where the enlarged arterial feeder connected to the ectatic vein or level of the AVM nidus. The lateral epidural AVS was determined by identifying the vertebral level of the fistula draining vein (near the neural foramen) that connects to the dilated perimedullary venous plexi. ${ }^{6}$ The ventral and dorsal epidural AVSs were determined by identifying the vertebral level of the arterialized spinal epidural venous plexus seen on the first-phase spinal MRA. 

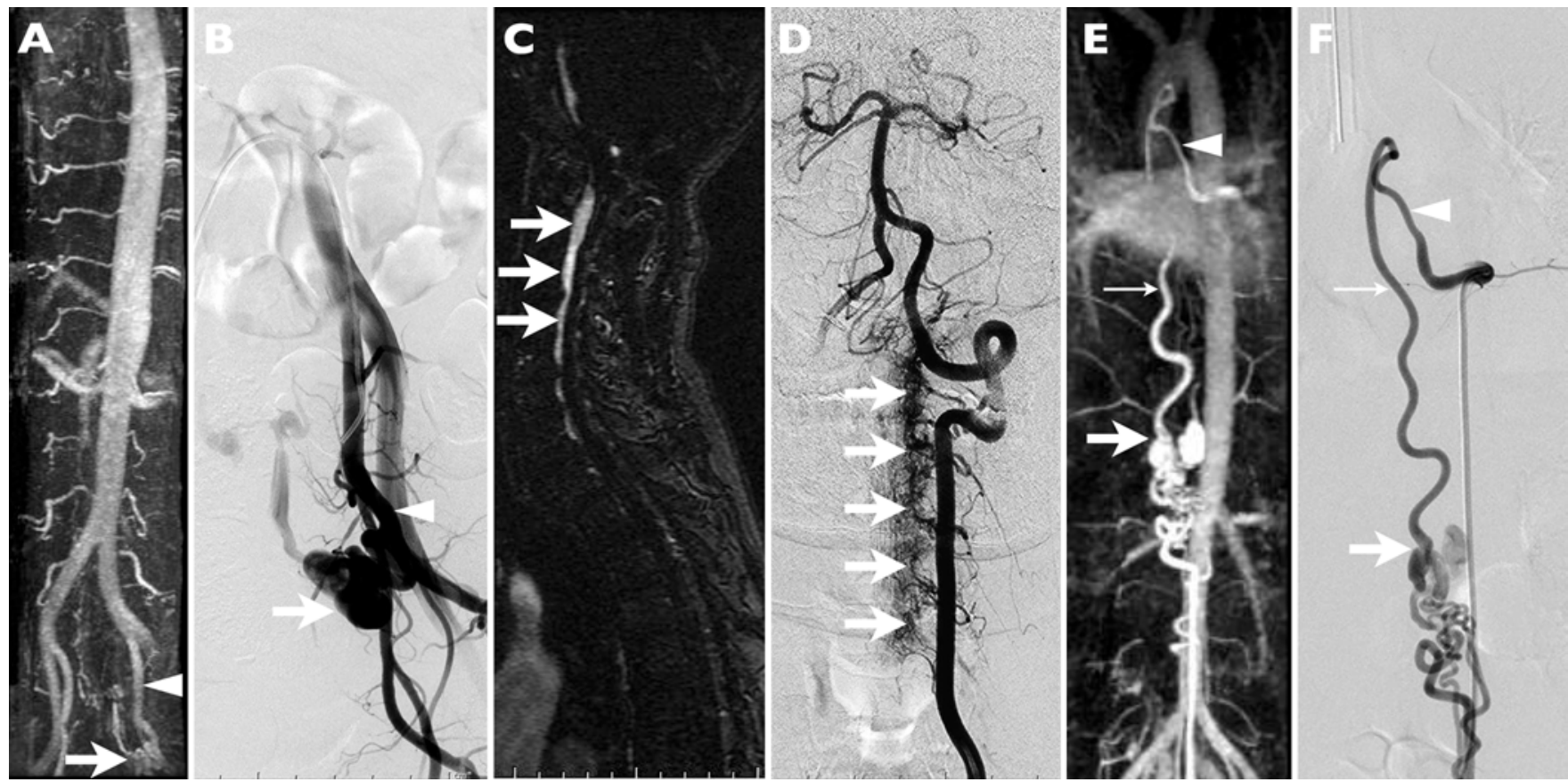

FIG. 1. Paraspinal AVS on coronal reconstruction MIP MR angiogram (A) and a left internal iliac angiogram (B) showing the direct communication of the enlarge left internal iliac artery (arrowhead) with an ectatic paravertebral vein (arrow). Spinal dural (ventral epidural) AVS on sagittal reconstruction MIP MR angiogram (C) shows the arterialization of the ventral epidural venous plexus (arrows), which corresponds with the venous receptacle of the AVS seen on the left vertebral angiogram (D, arrows). Intradural fistulous-type AVS on coronal reconstruction MIP MR angiogram (E) and left T-7 intercostal angiogram (F) showing the enlargement of the anterior spinal artery (thin arrow), the radiculomedullary artery (arrowhead) from the left T-7 intercostal artery, and the direct communication of the anterior spinal artery with an ectatic perimedullary vein. The arrow points at the fistulous area.

\section{Correct Shunt Localization}

Correct shunt localization on MRA was considered when not more than 1 vertebral level difference above or below was noted in comparison with the shunt level documented on catheter angiography. The dominant arterial feeder was defined as the largest arterial feeder of the AVS.

\section{Catheter Spinal Angiography}

Within 2 weeks after MRA, all patients underwent catheter spinal angiography using a flat panel, biplane, vascular imaging system (Infinix VF-i/BP; Toshiba Medical Systems). The procedure was performed under general anesthesia with transient apnea during contrast injection. Multiple selective arterial injections were performed to detect the presence of spinal AVS, the vertebral level of the AVS, the spinal cord, and the shunt's arterial supply.

\section{Statistical Method}

The Stata program (version 11.0) was used to analyze the sensitivity, specificity, and accuracy of the spinal MRA.

\section{Results \\ Participants}

There were 20 spinal AVS patients during the study period: 6 patients with paraspinal AVS, 6 patients with spinal dural AVS, and 8 patients with intradural AVS. Five patients, all with the diagnosis of vertebrovertebral fistula, were excluded because CT angiography was used as the diagnostic tool instead of spinal MRA. Fifteen patients who received 20 spinal MRA and catheter angiography studies were included for image interpretation. Fourteen studies were pretherapeutic studies, and 6 studies were follow-up studies after treatment. The demographic characteristics, presentations, shunt locations, and the accuracy of spinal MRA for detecting and evaluating spinal AVS are summarized in Table 1.

\section{Detection of Spinal AVS}

Spinal MRA was able to demonstrate the arterialized draining vein during the first-phase scan in all 17 studies with AVS confirmed by catheter angiography. There was no AVS in 3 posttreatment follow-up studies in which spinal MRA showed a true negative result.

\section{AVS Subtype Discrimination and Shunt Localization}

Using our criteria, spinal MRA was able to correctly classify the spinal AVS subtype and locate the shunting level in 16 of 17 studies (94.1\%). An erroneously diagnosed case was a perimedullary micro-AVF, which was diagnosed as a spinal dural AVS because MRA was unable to differentiate the enlarged spinal feeding artery from the arterialized perimedullary veins.

\section{Detection of the Dominant Arterial Feeder}

The AVS dominant arterial feeder was visualized by spinal MRA in 9 of 17 studies on AVS (52.9\%). MRA was 

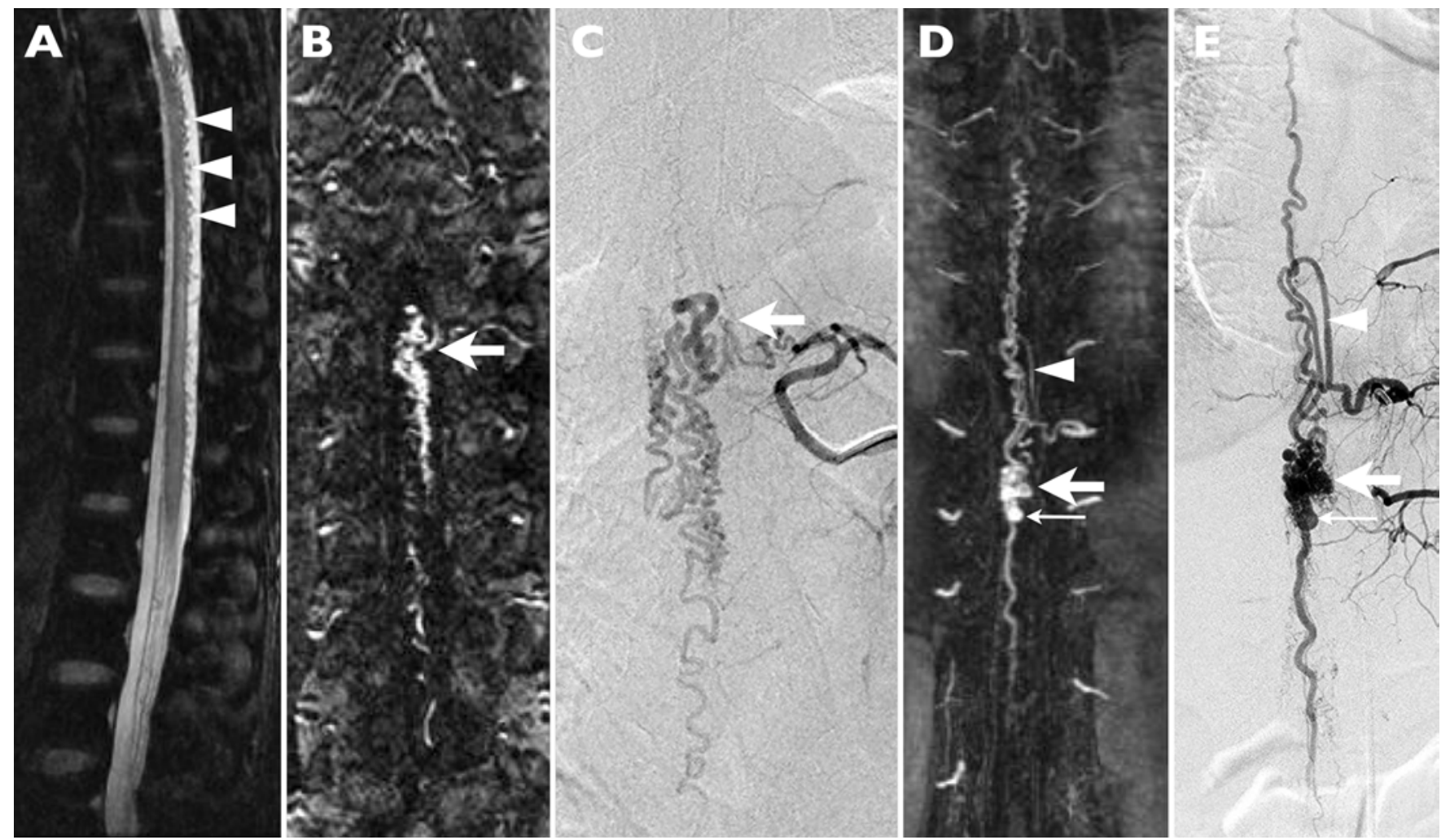

FIG. 2. A: Spinal dural (lateral epidural) AVS on a midsagittal T2-weighted image with fat suppression showing a T2 hyperintense signal within the thoracic cord and conus medullaris with serpiginous voids along the spinal cord dorsal aspect (arrowheads). B: Coronal reconstruction MIP MR angiogram showing the arterialization of the perimedullary venous plexus with a vein (arrow) connecting the venous plexus to the left T-6 neural foramen. C: Left T-6 intercostal angiogram demonstrates a fistulous area (arrow) and venous drainage of the AVS into the dilated perimedullary venous plexus. D: Intradural nidal-type AVS on coronal reconstruction MIP MR angiogram shows a tangled tortuous vascular network (AVM nidus) at the T9-10 spinal level (arrow), an intranidal aneurysm (thin arrow), and an enlarged radiculomedullary artery (arrowhead) from the left T-9 intercostal artery. E: Left T-9 intercostal angiogram showing findings similar to those of MR angiogram.

unable to demonstrate the dominant arterial feeder in all cases with ventral epidural dural AVS or posttreatment residual dural AVS, 1 case of spinal dural AVS with arterial supply from the lateral spinal artery, 1 case of thoracic cord AVM, and 1 case of perimedullary micro-AVF.

The sensitivity, specificity, accuracy, positive predictive value, and negative predictive value of spinal MRA for detecting spinal AVS and the dominant arterial feeder, discriminating the AVS subtype, and localizing the shunting area are summarized in Table 2.

\section{Discussion}

A total of 20 spinal AVS cases were collected during the 4.5-year study period, with a close distribution of numbers between subtypes. Intradural AVS was the most common subtype in our series, not spinal dural AVS as stated in previously reported studies. ${ }^{15,17,25}$

Many spinal MRA techniques have been proposed for detecting the normal spinal cord vessel and evaluating spinal AVS. Contrast-enhanced time-of-flight provides adequate spatial resolution for evaluating the spinal cord vessel. Due to its long acquisition time and lack of temporal resolution, only the large spinal cord veins in normal subjects and spinal AVS patients can be demonstrated. ${ }^{5,6}$
The 2D and 3D phase-contrast MRA techniques using a maximum encoding velocity of 20 to $30 \mathrm{~cm} / \mathrm{second}$ for high-flow spinal AVS, or 6 to $10 \mathrm{~cm} / \mathrm{second}$ for lowflow spinal AVS, and the injection of an intravenous MR contrast medium have been shown to demonstrate the enlarged arterial feeders of the high-flow spinal AVS and enlarged veins in cases with spinal AVS. The limitations of this technique are the lack of temporal resolution and the difficulty in selecting the optimal encoding velocity for each spinal AVS. ${ }^{23,24,29}$

Time-resolved spinal MRA is a new MRA technique that combines fast multiphase MRA with parallel imaging to improve the temporal resolution (3-7 seconds) in exchange for image spatial resolution and signal-to-noise ratio. This technique was able to identify and localize spinal AVS on 1.5-T MRA and also demonstrate the artery of Adamkiewicz on 3-T MRA., 1,4,16,33

Currently, the most commonly used MRA technique for detecting and evaluating spinal AVS is fast 3D contrast-enhanced MRA using elliptic centric $k$-space encoding with an acquisition time less than 1 minute/phase and spatial resolution adequate for demonstrating pathological intradural vessels. 2,27

Our spinal MRA technique has 2 different technical aspects, which are 1) the use of hand syringe contrast in- 
TABLE 1. The participants' demographic characteristics, presentations, shunt locations, and accuracy of spinal MRA for detecting and evaluating spinal AVS

\begin{tabular}{|c|c|c|c|c|c|c|c|}
\hline Patient No. \& Type of AVS & Sex/Age & Presentation & $\begin{array}{l}\text { Detection of } \\
\text { AVS by MRA }\end{array}$ & $\begin{array}{c}\text { Shunt Location } \\
\text { on DSA }\end{array}$ & $\begin{array}{l}\text { Correct Shunt } \\
\text { Level on MRA }\end{array}$ & $\begin{array}{l}\text { Correct Subtype } \\
\text { on MRA }\end{array}$ & $\begin{array}{l}\text { Correct Dominan } \\
\text { Feeder on MRA }\end{array}$ \\
\hline \multicolumn{8}{|l|}{ Paraspinal AVS ( $n=1)$} \\
\hline 1 & $\mathrm{M} / 46 \mathrm{yrs}$ & Rt foot paresthesia & Yes & S2-3 & Yes & Yes & Yes \\
\hline \multicolumn{8}{|l|}{ Spinal dural AVS $(n=6)$} \\
\hline \multicolumn{8}{|l|}{ Ventral epidural group } \\
\hline 2 & $\mathrm{M} / 44 \mathrm{yrs}$ & Asymptomatic & Yes & C2-6 & Yes & Yes & No \\
\hline 3 & $\mathrm{~F} / 51 \mathrm{yrs}$ & Tinnitus & Yes & C-2 & Yes & Yes & No \\
\hline \multicolumn{8}{|l|}{ Lateral epidural group } \\
\hline 4 & $M / 38$ yrs & Myelopathy & Yes & T-6 & Yes & Yes & Yes \\
\hline 5 & M/65 yrs & Myelopathy & Yes & $\mathrm{T}-10$ & Yes & Yes & Yes \\
\hline 6 & M/69 yrs & Myelopathy & Yes & L4-5 & Yes & Yes & No \\
\hline 7 & $\mathrm{M} / 47 \mathrm{yrs}$ & Myelopathy & Yes & T-6 & Yes & Yes & Yes \\
\hline \multicolumn{8}{|l|}{ Intradural AVS $(n=7)$} \\
\hline \multicolumn{8}{|l|}{ Glomerular type } \\
\hline 8 & $\mathrm{~F} / 25 \mathrm{yrs}$ & Hematomyelia & Yes & $\mathrm{T}-10$ & Yes & Yes & Yes \\
\hline 9 & $\mathrm{M} / 15 \mathrm{yrs}$ & Myelopathy & Yes & $\mathrm{T}-1$ & Yes & Yes & No \\
\hline 10 & $\mathrm{M} / 15 \mathrm{yrs}$ & $\mathrm{SAH}$ & Yes & $\mathrm{C} 7-\mathrm{T} 2$ & Yes & Yes & Yes \\
\hline 11 & $\mathrm{~F} / 13 \mathrm{yrs}$ & Hematomyelia & Yes & T9-10 & Yes & Yes & Yes \\
\hline \multicolumn{8}{|l|}{ Fistulous type } \\
\hline 12 & $\mathrm{~F} / 25 \mathrm{yrs}$ & Hematomyelia & Yes & $\mathrm{C}-2$ & Yes & Yes & Yes \\
\hline 13 & $\mathrm{~F} / 25 \mathrm{yrs}$ & Myelopathy & Yes & $\mathrm{T}-12$ & No & No & No \\
\hline 14 & $\mathrm{M} / 8 \mathrm{mos}$ & Hematomyelia & Yes & $\mathrm{T}-12$ & Yes & Yes & Yes \\
\hline \multicolumn{8}{|l|}{ Posttreatment FU $(n=6)$} \\
\hline \multicolumn{8}{|l|}{ Spinal dural AVS } \\
\hline 15 & $\mathrm{M} / 38 \mathrm{yrs}$ & FU & Yes & $\mathrm{T}-6$ & Yes & Yes & No \\
\hline 16 & M/65 yrs & FU & Yes & $\mathrm{T}-10$ & Yes & Yes & No \\
\hline 17 & $M / 38$ yrs & FU & Yes & $\mathrm{T}-5$ & Yes & Yes & No \\
\hline 18 & $\mathrm{M} / 47 \mathrm{yrs}$ & FU & No & No AVS & No AVS & No AVS & No AVS \\
\hline \multicolumn{8}{|l|}{ Intradural AVS } \\
\hline 19 & $\mathrm{~F} / 56 \mathrm{yrs}$ & FU & No & No AVS & No AVS & No AVS & No AVS \\
\hline 20 & $\mathrm{~F} / 25 \mathrm{yrs}$ & FU & No & No AVS & No AVS & No AVS & No AVS \\
\hline
\end{tabular}

DSA = digital subtraction angiography; FU = follow-up; $\mathrm{SAH}=$ subarachnoid hemorrhage.

jection instead of an automatic contrast injector machine and 2) the dose of the gadolinium-based contrast agent lowered to $0.15 \mathrm{mmol} / \mathrm{kg}$. The rationale for choosing the MR contrast agent and injection dose are based on the fact that gadobenate dimeglumine's relaxivity for shortening T1-weighted signals is twice as high as those of other MRI contrast agents at $0.5 \mathrm{mmol} / \mathrm{ml}$, and the standard dose of $0.1 \mathrm{mmol} / \mathrm{kg}$ body weight is sufficient for performing MRA on the cerebral vessels. ${ }^{28}$ Because spinal vessels are much smaller than cerebral vessels, we increased the dose of the contrast agent to $0.15 \mathrm{mmol} / \mathrm{kg}$ body weight in order to increase the contrast-to-noise ratio of the spinal vasculature. The reasons why we did not increase the dose to its maximum dose of $0.2 \mathrm{mmol} / \mathrm{kg}$ were to lower the investigation costs and the risk of nephrogenic systemic fibrosis, which increases with higher doses of the contrast agent. The low viscosity and the small amount of gadoliniumbased contrast agent used led us to initiate the administra-

TABLE 2. Sensitivity, specificity, accuracy, PPV, and NPV of spinal MRA for detecting and evaluating spinal AVS

\begin{tabular}{lccccc}
\hline \multicolumn{1}{c}{ Variable } & Sensitivity $(95 \% \mathrm{Cl})$ & Specificity $(95 \% \mathrm{Cl})$ & Accuracy $(95 \% \mathrm{Cl})$ & $\mathrm{PPV}(95 \% \mathrm{Cl})$ & $\mathrm{NPV}(95 \% \mathrm{Cl})$ \\
\hline Detection of AVS & $100(100.0-100.0)$ & $100(100.0-100.0)$ & $100(100.0-100.0)$ & $100(100.0-100.0)$ & $100(100.0-100.0)$ \\
\hline Subtype discrimination & $94.12(83.81-104.43)$ & $100(100.0-100.0)$ & $97.06(91.29-100.0)$ & $100(100.0-100.0)$ & $75(56.02-93.98)$ \\
\hline Shunt localization & $88.24(74.11-102.36)$ & $100(100.0-100.0)$ & $94.12(86.22-100.0)$ & $100(100.0-100.0)$ & $60(38.53-81.47)$ \\
\hline Dominant feeder detection & $52.94(31.07-74.82)$ & $100(100.0-100.0)$ & $76.47(64.24-88.7)$ & $100(100.0-100.0)$ & $27.27(7.75-46.79)$ \\
\hline
\end{tabular}

$\mathrm{NPV}=$ negative predictive value; $\mathrm{PPV}=$ positive predictive value. 
tion of contrast with our hand syringe technique in order to lower the expense caused by the disposable equipment. We also used hand syringe contrast injection for all pediatric MRA cases and, in the case of an intravenous catheters smaller than 20 gauge, in order to reduce the risk of contrast media leakage. The purpose for studying our hand contrast injection technique was to show that spinal MRA could be easily done at all MRI centers with acceptable image quality despite not having an automatic injector machine.

Previous studies on fast 3D contrast-enhanced MRA have shown variable rates in the detection and localization of spinal AVS. The largest spinal MRA series using the fast 3D contrast-enhanced MRA technique was by Mull et al., ${ }^{26}$ in which the presence of spinal AVS was detected in all 31 cases and shunt level localization was correct in 19 cases of spinal dural AVS, with the exception of 1 tentorial dural AVS case with caudal spinal venous drainage.

This study showed a $100 \%$ sensitivity and specificity rate for the detection of the presence of spinal AVS with no false-positive or false-negative results. Various subtypes of spinal AVS were included to study the accuracy of the diagnostic criteria for subtype discrimination, which incorporated both spinal MRI and MRA findings. Our spinal MRA and diagnostic criteria demonstrated 97\% accuracy for differentiating each subtype of spinal AVS, with the exception in the perimedullary micro-AVF subtype. In our opinion, this exception was caused by the limitations of MRA's spatial and temporal resolution to separate small arterial feeders from the much larger arterialized draining veins. Our MRA protocol limited the spatial and temporal resolution, as shown by the $52.9 \%$ detection rate of the shunt's dominant arterial feeder and the low detection rate of the artery of Adamkiewicz. Using a larger amount of contrast agent, or a larger bolus and faster constant injection rate by an injector machine, will improve the contrast-to-noise ratio of the MRA images, which will help improve the spatial resolution of the image and yield a better detection rate of the shunt arterial feeder. However, to further improve the detection of the shunt's dominant arterial feeder, we needed to decrease the acquisition time in order to improve the image's temporal resolution so an earlier enhancing artery could be differentiated from the vein. Because our MRA technique used 3D contrast-enhanced MRA, which has a limited temporal resolution, the detection of the shunt's dominant artery feeder will still be a limitation of this technique despite improvements in the image's spatial resolution. Future improvements in both MRA's spatial and temporal resolution, or better postprocessing image reconstruction techniques, are needed to increase the detection rate of these small arteries, which provide the important data needed for guiding catheter spinal angiography. It should be stressed that despite the high accuracy rate of spinal MRA in our study, the statistical power of the results was still insufficient due to the small number of spinal AVS samples. Therefore, a larger sample size is needed in the future to boost the statistical power of this study. Currently, catheter spinal angiography is still required for the complete evaluation and follow-up of spinal AVS at our institution. To the best of our knowledge, this study contained the largest number of posttreatment follow-up cases where spinal MRA showed a $100 \%$ accuracy rate for the detection of residual AVS. In the future, if a larger posttreatment follow-up population is obtained, we could avoid performing catheter spinal angiography on cases with negative spinal MRA.

\section{Conclusions}

We showed that limiting the use of contrast media, not using an automatic injector machine, and using our fast 3D contrast-enhanced MRA technique with manual syringe contrast injection can accurately detect the presence of spinal AVS, locate the shunt level, and discriminate the AVS subtype in most cases, but is limited when detecting small arterial feeders.

\section{Acknowledgments}

We thank Dr. Apichat Tantraworasin for his consultations about the statistical analysis and providing encouragement throughout this difficult project.

\section{References}

1. Ali S, Cashen TA, Carroll TJ, McComb E, Muzaffar M, Shaibani A, et al: Time-resolved spinal MR angiography: initial clinical experience in the evaluation of spinal arteriovenous shunts. AJNR Am J Neuroradiol 28:1806-1810, 2007

2. Backes WH, Nijenhuis RJ: Advances in spinal cord MR angiography. AJNR Am J Neuroradiol 29:619-631, 2008

3. Bao YH, Ling F: Classification and therapeutic modalities of spinal vascular malformations in 80 patients. Neurosurgery 40:75-81, 1997

4. Bley TA, Duffek CC, François CJ, Schiebler ML, Acher CW, Mell M, et al: Presurgical localization of the artery of Adamkiewicz with time-resolved 3.0-T MR angiography. Radiology 255:873-881, 2010

5. Bowen BC, DePrima S, Pattany PM, Marcillo A, Madsen P, Quencer RM: MR angiography of normal intradural vessels of the thoracolumbar spine. AJNR Am J Neuroradiol 17:483-494, 1996

6. Bowen BC, Fraser K, Kochan JP, Pattany PM, Green BA, Quencer RM: Spinal dural arteriovenous fistulas: evaluation with MR angiography. AJNR Am J Neuroradiol 16:20292043, 1995

7. Cho WS, Kim KJ, Kwon OK, Kim CH, Kim J, Han MH, et al: Clinical features and treatment outcomes of the spinal arteriovenous fistulas and malformation: clinical article. J Neurosurg Spine 19:207-216, 2013

8. Chuang NA, Shroff MM, Willinsky RA, Drake JM, Dirks PB, Armstrong DC: Slow-flow spinal epidural AVF with venous ectasias: two pediatric case reports. AJNR Am J Neuroradiol 24:1901-1905, 2003

9. Chul Suh D, Gon Choi C, Bo Sung K, Kim KK, Chul Rhim S: Spinal osseous epidural arteriovenous fistula with multiple small arterial feeders converging to a round fistular nidus as a target of venous approach. AJNR Am J Neuroradiol 25:69-73, 2004

10. Cognard C, Semaan H, Bakchine S, Miaux Y, Thibault S, Sola Martinez MT, et al: Paraspinal arteriovenous fistula with perimedullary venous drainage. AJNR Am J Neuroradiol 16:2044-2048, 1995

11. da Costa L, Dehdashti AR, terBrugge KG: Spinal cord vascular shunts: spinal cord vascular malformations and dural arteriovenous fistulas. Neurosurg Focus 26(1):E6, 2009

12. Dabus G, Nimmagadda A, Russell EJ: Cervical epidural arteriovenous fistula presenting with radiculopathy: transvenous 
embolization using Onyx. Interv Neuroradiol 17:380-385, 2011

13. Geibprasert S, Pereira V, Krings T, Jiarakongmun P, Toulgoat F, Pongpech S, et al: Dural arteriovenous shunts: a new classification of craniospinal epidural venous anatomical bases and clinical correlations. Stroke 39:2783-2794, 2008

14. Goyal M, Willinsky R, Montanera W, terBrugge K: Paravertebral arteriovenous malformations with epidural drainage: clinical spectrum, imaging features, and results of treatment. AJNR Am J Neuroradiol 20:749-755, 1999

15. Jahan RVF: Vascular anatomy, pathophysiology, and classification of vascular malformations of the spinal cord. Seminars in Cerebrovascular Diseases and Stroke 2:186-200, 2002

16. Jaspers K, Nijenhuis RJ, Backes WH: Differentiation of spinal cord arteries and veins by time-resolved MR angiography. J Magn Reson Imaging 26:31-40, 2007

17. Kendall BE, Logue V: Spinal epidural angiomatous malformations draining into intrathecal veins. Neuroradiology 13:181-189, 1977

18. Kiyosue H, Tanoue S, Okahara M, Hori Y, Kashiwagi J, Mori H: Spinal ventral epidural arteriovenous fistulas of the lumbar spine: angioarchitecture and endovascular treatment. Neuroradiology 55:327-336, 2013

19. Krings T, Geibprasert S: Spinal dural arteriovenous fistulas. AJNR Am J Neuroradiol 30:639-648, 2009

20. Krings T, Mull M, Gilsbach JM, Thron A: Spinal vascular malformations. Eur Radiol 15:267-278, 2005

21. Lim SM, Choi IS: Spinal epidural arteriovenous fistula: a unique pathway into the perimedullary vein. A case report. Interv Neuroradiol 15:466-469, 2009

22. Luetmer PH, Lane JI, Gilbertson JR, Bernstein MA, Huston J III, Atkinson JL: Preangiographic evaluation of spinal dural arteriovenous fistulas with elliptic centric contrast-enhanced MR Angiography and effect on radiation dose and volume of iodinated contrast material. AJNR Am J Neuroradiol 26:711-718, 2005

23. Mascalchi M, Bianchi MC, Quilici N, Mangiafico S, Ferrito G, Padolecchia R, et al: MR angiography of spinal vascular malformations. AJNR Am J Neuroradiol 16:289-297, 1995

24. Mascalchi M, Quilici N, Ferrito G, Mangiafico S, Scazzeri F, Torselli P, et al: Identification of the feeding arteries of spinal vascular lesions via phase-contrast MR angiography with three-dimensional acquisition and phase display. AJNR Am J Neuroradiol 18:351-358, 1997

25. Merland JJ, Riche MC, Chiras J: Intraspinal extramedullary arteriovenous fistulae draining into the medullary veins. J Neuroradiol 7:271-320, 1980

26. Mull M, Nijenhuis RJ, Backes WH, Krings T, Wilmink JT, Thron A: Value and limitations of contrast-enhanced MR angiography in spinal arteriovenous malformations and dural arteriovenous fistulas. AJNR Am J Neuroradiol 28:12491258, 2007

27. Pattany PM, Saraf-Lavi E, Bowen BC: MR angiography of the spine and spinal cord. Top Magn Reson Imaging 14:444-460, 2003

28. Pintaske J, Martirosian P, Graf H, Erb G, Lodemann KP, Claussen CD, et al: Relaxivity of Gadopentetate Dimeglumine (Magnevist), Gadobutrol (Gadovist), and Gadobenate
Dimeglumine (MultiHance) in human blood plasma at 0.2, 1.5, and 3 Tesla. Invest Radiol 41:213-221, 2006

29. Provenzale JM, Tien RD, Felsberg GJ, Hacein-Bey L: Spinal dural arteriovenous fistula: demonstration using phase contrast MRA. J Comput Assist Tomogr 18:811-814, 1994

30. Rodesch G, Hurth M, Alvarez H, Ducot B, Tadie M, Lasjaunias P: Angio-architecture of spinal cord arteriovenous shunts at presentation. Clinical correlations in adults and children. The Bicêtre experience on 155 consecutive patients seen between 1981-1999. Acta Neurochir (Wien) 146:217227, 2004

31. Rodesch G, Hurth M, Alvarez H, Tadié M, Lasjaunias P: Classification of spinal cord arteriovenous shunts: proposal for a reappraisal--the Bicêtre experience with 155 consecutive patients treated between 1981 and 1999. Neurosurgery 51:374-380, 2002

32. Rodesch G, Lasjaunias P: Spinal cord arteriovenous shunts: from imaging to management. Eur J Radiol 46:221-232, 2003

33. Saindane AM, Boddu SR, Tong FC, Dehkharghani S, Dion JE: Contrast-enhanced time-resolved MRA for pre-angiographic evaluation of suspected spinal dural arterial venous fistulas. J Neurointerv Surg 7:135-140, 2015

34. Spetzler RF, Detwiler PW, Riina HA, Porter RW: Modified classification of spinal cord vascular lesions. J Neurosurg 96 (2 Suppl):145-156, 2002

35. Toi H, Matsubara S, Watanabe S, Yamashita T, Uno M: Paraspinal arteriovenous fistula presenting with subarachnoid hemorrhage and acute progressive myelopathy-case report. Neurol Med Chir (Tokyo) 51:846-849, 2011

\section{Disclosures}

The authors report no conflict of interest concerning the materials or methods used in this study or the findings specified in this paper.

\section{Author Contributions}

Conception and design: Unsrisong. Acquisition of data: Unsrisong, Taphey. Analysis and interpretation of data: Unsrisong. Drafting the article: Unsrisong. Critically revising the article: Unsrisong. Reviewed submitted version of manuscript: Unsrisong, Oranratanachai. Approved the final version of the manuscript on behalf of all authors: Unsrisong. Statistical analysis: Unsrisong. Administrative/technical/material support: Taphey. Study supervision: Oranratanachai.

\section{Supplemental Information \\ Previous Presentations}

Portions of this work were presented as a poster at the 10th meeting of Asian Australasian Federation of Interventional and Therapeutic Neuroradiology, Nagoya, Japan, June 14, 2012.

\section{Correspondence}

Kittisak Unsrisong, Department of Radiology, Maharaj Nakorn Chiang Mai Hospital, Chiang Mai University, 110 Suthep Rd., Chiangmai 50200, Thailand. email: kitlight@ hotmail.com. 